\title{
New strategies for the treatment of lysosomal storage diseases (Review)
}

\author{
GIANCARLO PARENTI, CLAUDIO PIGNATA, PIETRO VAJRO and MARIACAROLINA SALERNO \\ Department of Pediatrics, Federico II University, Naples, Italy
}

Received September 4, 2012; Accepted October 29, 2012

DOI: $10.3892 /$ ijmm.2012.1187

\begin{abstract}
The lysosomal storage diseases (LSDs) are a group of inherited metabolic disorders caused by the deficiency of any of the lysosomal functions, in most cases of lysosomal hydrolases. LSDs are typically characterized by storage of a variety of substrates in multiple tissues and organs and by the variable association of unusual clinical manifestations that are often responsible for physical and neurological handicaps. During the past two decades, research in the field of LSDs has made marked progress, particularly with the development of a variety of innovative therapeutic approaches. These include several strategies aimed at increasing the residual activity of the missing enzyme, such as hematopoietic stem cell transplantation, enzyme replacement therapy, pharmacological chaperone therapy and gene therapy. An alternative approach is based on reducing the synthesis of the stored substrate. More recently, the improved knowledge on LSD pathophysiology has indicated additional targets of therapy. The recent progress made in the treatment of LSDs represents a good model that may be extended to other genetic disorders.
\end{abstract}

\section{Contents}

1. Introduction

2. Advances in the understanding of LSD pathophysiology and their therapeutic implications

3. Hematopoietic stem cell transplantation

4. Enzyme replacement therapy

5. Small molecule pharmacological chaperones

6. Combination of chaperones and ERT

7. Proteostasis regulators

8. Gene therapy

9. Substrate reduction therapy

10. Other experimental approaches

11. Conclusion

Correspondence to: Professor Giancarlo Parenti, Department of Pediatrics, Federico II University, Via S. Pansini 5, I-80131 Naples, Italy E-mail: parenti@unina.it

Key words: lysosomal storage disease, enzyme replacement therapy, pharmacological chaperone therapy, gene therapy

\section{Introduction}

The lysosomal storage diseases (LSDs) are a group of inherited metabolic disorders caused by mutations in genes encoding soluble lysosomal hydrolases, integral membrane proteins and transporters (1). The defective function of these proteins results in the impaired intracellular turnover and disposal of a broad range of complex molecules including sphingolipids, glycosaminoglycans, glycoproteins and glycogen.

The pathology of LSDs is typically characterized by intra-lysosomal storage of a variety of substrates in multiple tissues and organs. Thus, the phenotypes of these disorders are complex, and characterized by the variable association of visceral, ocular, hematologic, skeletal and neurological manifestations. These manifestations are in most instances responsible for physical and neurological handicaps. In particular, those related to the involvement of the central nervous system (CNS) may cause progressive neurodegeneration and severe cognitive impairment.

More than 40 LSDs are currently known and are traditionally classified according to the chemical properties of the accumulated substrate. Although each of these disorders is rare, their overall prevalence is relatively high, compared to other groups of rare diseases, and is estimated at 1 in 8,000 live births (2).

For their cumulative prevalence, for the debilitating effects of their clinical manifestations, and for the need of a multidisciplinary approach to the care of patients, LSDs represent a challenge for pediatricians and other physicians, and a heavy burden in terms of public health and economical costs.

An additional and attractive reason of interest for these disorders derives from the fact that during the past two decades the research in the field of LSDs has made significant progress, particularly with the development of a variety of innovative therapeutic approaches, that may be potentially extended to other genetic diseases (3).

The marked advancement in the treatment of LSDs has been largely stimulated by the improved knowledge on their molecular bases and pathophysiology. Further impulse to the development of therapies for LSDs has derived from the availability of technologies allowing large-scale production, purification and manipulation of new drugs, recombinant proteins and viral vectors, and from the orphan drug legislation, that encouraged biotech companies to invest in the treatments for rare diseases (4). 


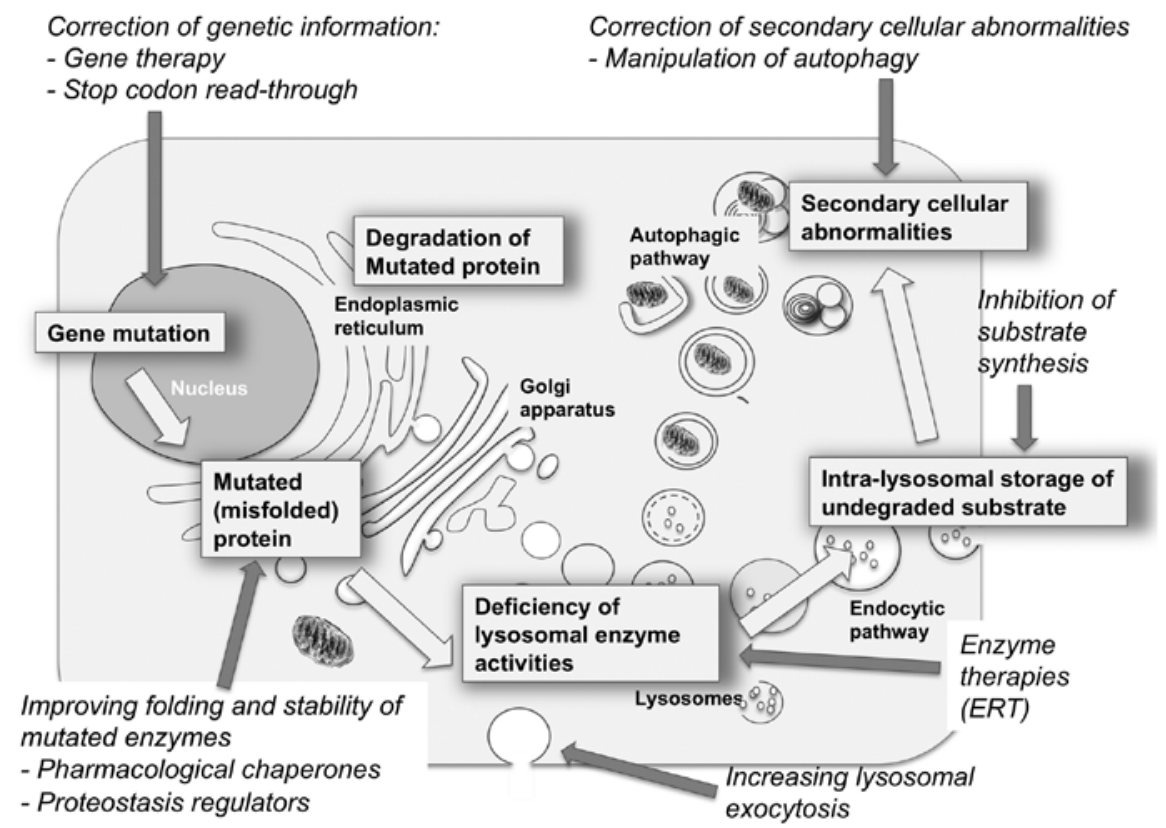

Figure 1. The pathogenetic cascade of LSDs (white boxes and white arrows). LSDs are due to mutations that cause the synthesis of mutated proteins and the defective activity of a lysosomal function. This results in intra-lysosomal storage of undegraded substrates and triggers secondary cellular abnormalities. Each of these events is a potential target of therapy, with different strategies and rationale (italics and grey arrows).

\section{Advances in the understanding of LSD pathophysiology and their therapeutic implications}

The understanding of LSD pathophysiology has rapidly evolved during the past decade and it is now clear that storage of substrates within the lysosomal compartment triggers a number of secondary cellular responses that eventually lead to cell death and tissue damage. Substrate storage acts as a primum movens that leads to the derangement of housekeeping cellular functions and pathways, including receptor activation by non-physiologic ligands, modulation of receptor responses and signal transduction cascades, activation of inflammatory responses, impaired intracellular trafficking of vesicles, membranes and membrane-bound proteins, impairment of autophagy, and others (5).

It is also becoming clear that each of the events in the pathogenetic cascade of LSDs is a potential target of therapy and that various approaches, based on different strategies and rationale, may be exploited to treat these disorders.

Until two decades ago, the treatment of patients affected by LSDs was exclusively based on palliative or supportive medical therapies. The first therapeutic strategies directed toward the correction of the basic defect of these disorders were introduced in the early 1990s, and since then a number of novel approaches have been proposed or tested in preclinical studies.

The most obvious approach to correct loss-of-function diseases, such as LSDs, caused in most cases by the deficiency of a lysosomal hydrolase, is to restore or replace the defective enzymatic activity. Thus, the majority of the approaches thus far developed to treat LSDs are aimed at increasing the cell and tissue levels of the missing enzyme. These include hematopoietic stem cell transplantation (HSCT), enzyme replacement therapy (ERT), pharmacological chaperone therapy (PCT) and gene therapy (GT).
These types of approaches may be particularly suitable for disorders such as LSDs, as it is assumed that clinical symptoms generally develop in patients when the level of residual enzyme falls below a critical threshold. Thus, residual activities greater than $10 \%$ of the normal level may, in principle, be sufficient to prevent storage, and it is possible to speculate that even minor increases of enzyme activity may translate into some clinical benefit for patients.

An alternative approach, substrate reduction therapy (SRT), is based on reducing the synthesis of the substrates stored in the lysosomes.

In recent years, with the improved knowledge on the pathophysiology of LSDs, other innovative therapeutic strategies have been proposed and are being evaluated in preclinical studies (Fig. 1).

\section{Hematopoietic stem cell transplantation}

HSCT was the first therapeutic approach introduced in the treatment of LSDs. Bone marrow has been traditionally the graft source for this procedure. However, in recent years, a number of patients have been treated with unrelated donor umbilical cord blood transplant, allowing rapid and increased access to transplantation (6).

HSCT relies on the use of hematopoietic stem cells derived from a healthy donor as a therapeutic agent. By this approach, a dual effect may be achieved (Fig. 2); the first is to repopulate specific tissues by the donor's healthy cells, and the second and key effect is related to the secretion of functional lysosomal hydrolases by the donor's cells in the extracellular space and into the blood circulation. The secreted normal enzyme may be taken up by the recipient cells and may cross-correct the enzyme defect in these cells.

The clinical experience obtained so far with HSCT, however, suggests that this procedure is limited to few 


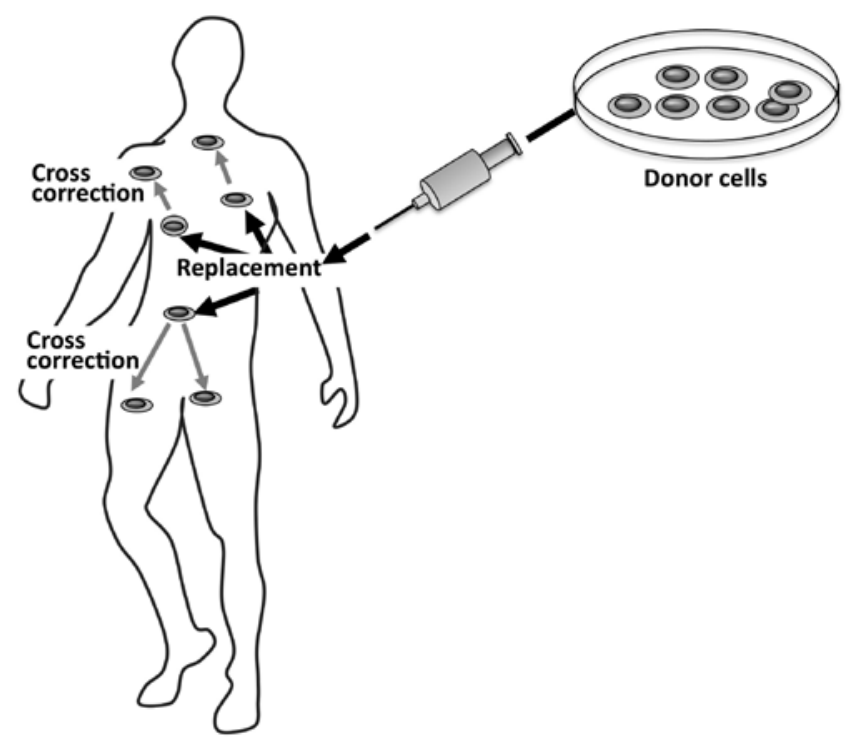

Figure 2. Hematopoietic stem cell transplantation is based on the use of hematopoietic stem cells derived from a healthy donor as a therapeutic agent. Once injected into patients the cells have a dual effect; one is to replace and repopulate specific tissues by the donor's healthy cells, and the second effect is related to the secretion of functional lysosomal hydrolases by the donor's cells in the extracellular space and into the blood circulation. The secreted normal enzyme may be taken up by the recipient cells and may cross-correct the enzyme defect in these cells.

lysosomal disorders. HSCT is indicated for the treatment of mucopolysaccharidosis (MPS) I (7) and has beneficial effects on the visceral manifestations of MPS VI, in pre-symptomatic or late-onset Krabbe disease, and in the attenuated forms of metachromatic leukodystrophy (8). For MPS I indications, optimal timing, safety and efficacy of HSCT have recently been reviewed by a European panel of specialists that participated in a modified Delphi process to develop consensus-based statements on this approach (9). HSCT is considered the preferred treatment for patients with severe MPS I diagnosed before the age of 2.5 years, and may be considered in individual patients with intermediate phenotypes if there is a suitable donor.

For Krabbe disease and metachromatic leukodystrophy, disease phenotype and the extent of disease at the time of transplantation are of fundamental importance in determining outcomes (10).

A significant advantage of HSCT is that, as donor-derived, enzyme-producing cells are able to migrate to the brain. This procedure has the potential to improve neurocognitive function and quality of life, particularly when performed early in the course of the disease. On the other hand, HSCT is burdened by a significant mortality related to the procedure, by the limited availability of suitable donors, by the limited number of disorders that can be treated by this approach and by insufficient engraftment and correction of pathology in some tissues, such as bone or heart.

\section{Enzyme replacement therapy}

The most important advancement and a major breakthrough in the treatment of LSDs is ERT. This approach is based on periodic intravenous infusions of human recombinant lysosomal enzymes produced and purified on large scale from different sources with recombinant DNA techniques. Once injected, the recombinant wild-type enzymes are distributed to tissues, internalized by cells and targeted to the lysosomal compartment, where they replace the defective enzyme.

The development of ERT is an excellent example of how progress in the knowledge of lysosomal biology may lead to the development of novel therapeutic approaches. The rationale of ERT evolved from pivotal studies on the molecular and cellular mechanisms implicated in the sorting of newly synthesized and secreted lysosomal enzymes. These studies showed that lysosomal hydrolases are delivered to lysosomes through the mannose or the mannose-6-phosphate receptor pathways and that these receptors are also expressed at the plasma membrane of cells (11). Thus, the lysosomal enzymes can be internalized by cells and follow the endocytic route to be correctly delivered to lysosomes, by binding to the receptors exposed on the cellular membrane (Fig. 3). Based on these studies, it has become evident that the same route could be exploited to deliver recombinant enzymes to defective cells and tissues and that LSDs represented the best model of inherited metabolic disorders that could be treated by providing the missing enzyme from external sources.

The first attempts to develop an ERT were made in Gaucher disease. After more than two decades of clinical experience with thousands of patients treated, the success of ERT has been clearly documented in this disease. ERT has been firmly proven to be effective in ameliorating the visceral, hematological and biochemical manifestations of Gaucher disease and has substantially improved patient quality of life.

The success of ERT in Gaucher disease $(12,13)$ stimulated the development of this approach for the treatment of the most prevalent lysosomal disorders, for which ERT is presently considered the standard of care.

In Fabry disease, a lysosomal disorder due to the deficiency of $\alpha$-galactosidase $\mathrm{A}$ and involving vascular endothelia, heart and the peripheral nervous system, beneficial effects have been documented on cardiac and renal functions, together 


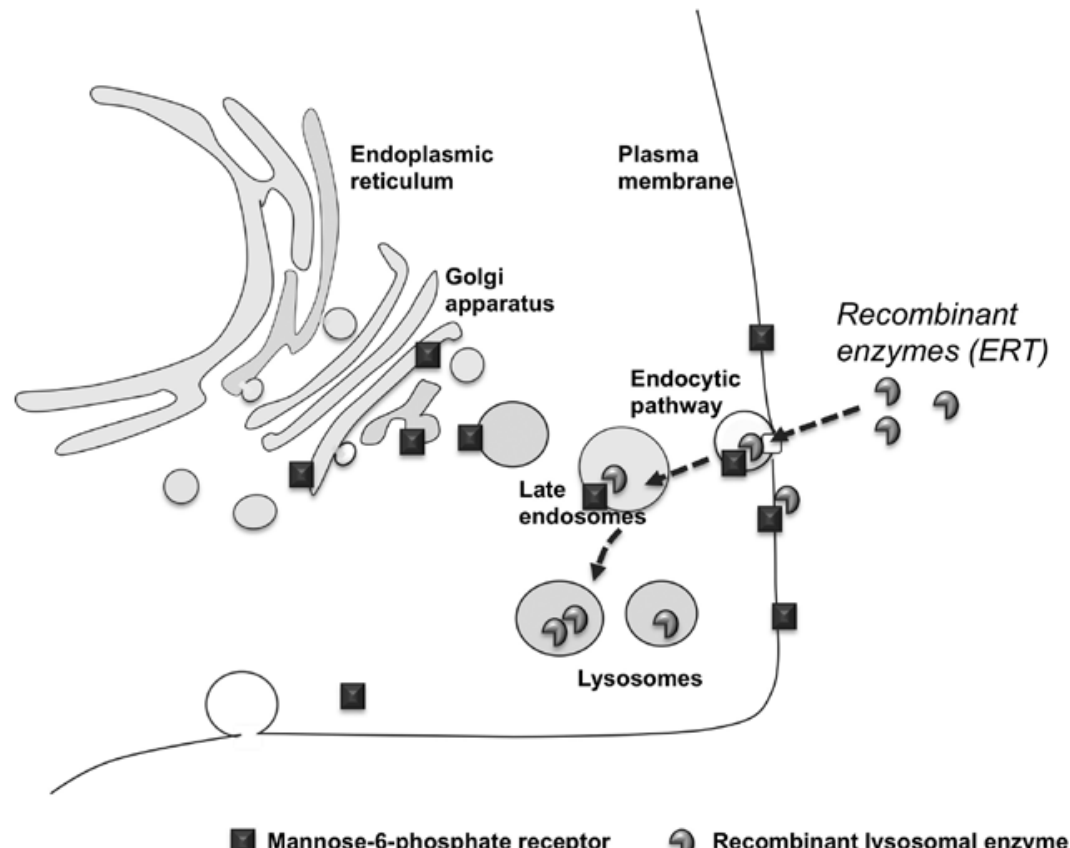

Figure 3. The rationale of ERT. ERT is based on periodic intravenous infusions of human recombinant lysosomal enzymes. The enzymes bind to the mannose or mannose-6-phosphate receptors available at the plasma membrane of cells. The enzymes are thus internalized by the cells and delivered to lysosomes through the endocytic route (dotted arrows).

with reduced pain and improved quality of life (14-16). Also, reduction of plasma and urinary levels of substrate documents ERT efficacy.

In Pompe disease, clinical experience has shown striking effects on cardiomyopathy, with reduced left ventricular mass index, prolonged survival in early onset patients (17), improved physical performance, stabilization or amelioration of skeletal muscle function in late onset patients $(18,19)$.

In MPS I, II and VI, the general benefits of ERT include improved walking ability, improved respiration and enhanced quality of life (7). A biochemical marker of ERT efficacy is the reduced urinary excretion of glycosaminoglycans observed in treated patients.

Due to the success of ERT in these disorders, recombinant enzymes are being tested to treat other LSDs, including MPS IV (Morquio disease), MPS VII (Sly disease), MPS IIIA (Sanfilippo disease A), metachromatic leukodystrophy and acid lipase deficiency.

On the other hand, clinical experience with ERT has shown that this approach has limitations, mostly related to the bioavailability of recombinant enzymes and to the high cost of therapies.

Recombinant enzymes are large molecules that do not freely diffuse across membranes and that are unable to reach therapeutic concentrations in some of the target tissues. Thus, a number of tissues remain refractory to this treatment and some patients experience limited clinical benefits under ERT treatment or continue to show signs of disease progression. Examples of the limitations of ERT are seen in MPS I, II and VI, where correction of pathology is insufficient in tissues such as bone, cartilage and heart, that are major sites of pathology. In Pompe disease, achieving corrective levels of $\alpha$-glucosidase activity and correcting pathology in skeletal muscles remains a major challenge (20).
Of even greater clinical relevance is the inability of recombinant enzymes to cross the blood-brain barrier (BBB) and reach the CNS $(21,22)$. As approximately two thirds of LSD patients have CNS involvement and progressive neurodegeneration, obtaining corrective enzyme levels in the brain is a major therapeutic goal.

To circumvent these problems and improve delivery of the enzymes to target tissues, including CNS, different strategies are being evaluated. All these strategies are still experimental.

Attempts to obtain corrective levels of enzyme activity in brain in neuronopathic LSDs have been made, by chemically manipulating the recombinant enzymes. $\beta$-glucuronidase, which is deficient in MPS VII, has been modified to increase the plasma half-life and facilitate its traffic through the BBB (23). Other approaches that are currently being explored are based on receptor-mediated penetration of $\mathrm{BBB}$, such as by coupling recombinant enzymes with transferrin and using transferrin receptor-mediated endocytosis for transport across the BBB (24).

In addition to these approaches an invasive procedure, such as intrathechal ERT administration, has been evaluated in preclinical studies for several lysosomal disorders and has been translated into human therapy for MPS I (25).

Attempts have also been made to improve muscle targeting of ERT in Pompe disease. Pompe disease is a metabolic myopathy due to the deficiency of $\alpha$-glucosidase. The recombinant $\alpha$-glucosidase (rhGAA) used for the treatment of this disorder has shown insufficient correction of pathology in skeletal muscles. Zhu et al (26) developed a glycoengineered rhGAA (neo-rhGAA) in which additional mannose 6-phosphate moieties were introduced that showed higher affinity for the mannose-6-phosphate receptor. In a Pompe disease mouse model, this modified enzyme promoted a greater clearance of lysosomal glycogen in muscles when compared to the 


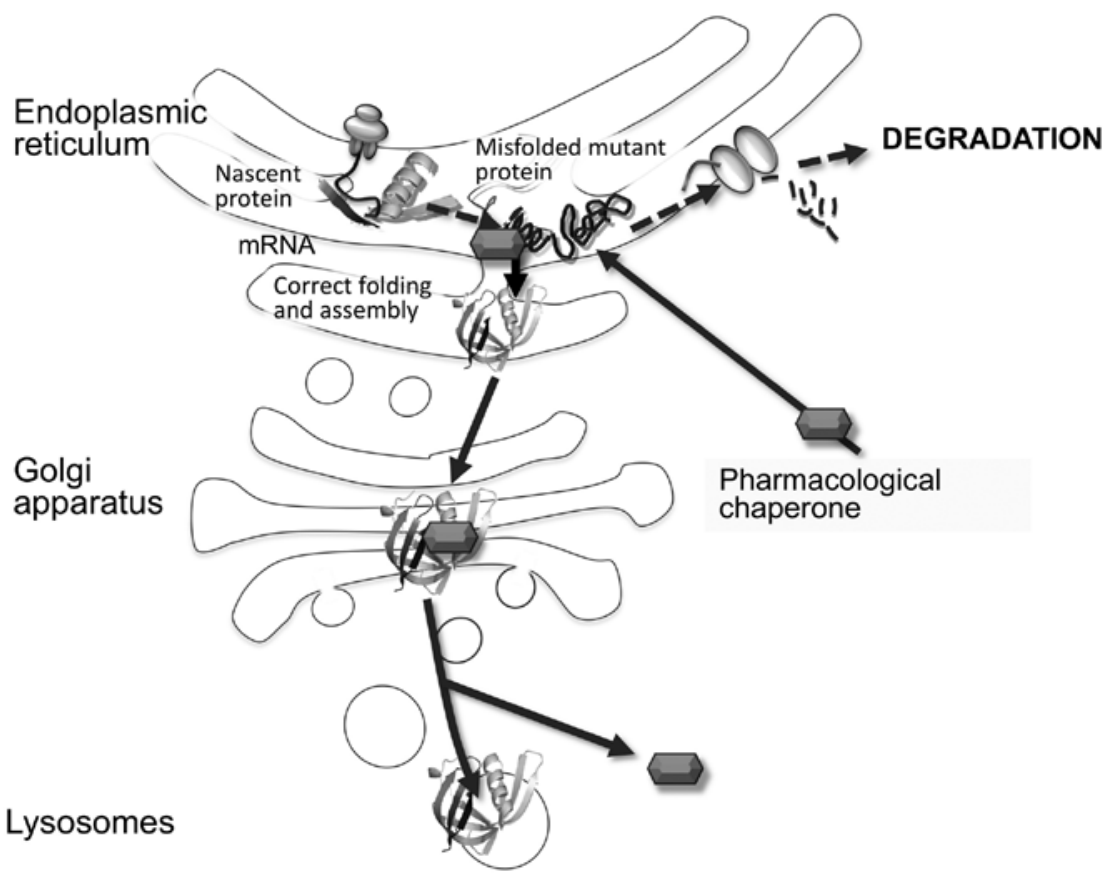

Figure 4. Pharmacological chaperone therapy is based on the concept that mutated misfolded proteins are recognized by the quality control systems of the endoplasmic reticulum and degraded. Small-molecule ligands (pharmacological chaperones) can interact with the mutant protein, favor its correct conformation, and enhance its stability. As a result, the enzymatic activity of the mutant protein is partially rescued.

unmodified enzyme. Other approaches are based on the fusion of a peptide tag derived from insulin-like growth factor-II (IGF-II), which is also a ligand for the mannose-6-phosphate receptor, onto rhGAA.

The high cost of ERT is another significant limitation and a major problem for the treatment of patients with LSDs. Initial investments in research and costs related to the production of large amounts of recombinant enzymes according to good manufacturing practice, contributed to the high cost of ERT. The treatment of a single LSD patient may require as much as several hundred thousand euros (or dollars) per year. Such high costs, that are acceptable in western countries, are barely affordable in underdeveloped countries, and limit the access of patients to ERT. The availability of novel molecular technologies may allow the production of less expensive enzyme preparations. As an example, recombinant $\beta$-glucosidase, the enzyme deficient in Gaucher disease, has been manufactured in plants or plant-derived cells (27).

\section{Small molecule pharmacological chaperones}

An approach that has recently gained much attention for the treatment of diseases due to protein misfolding in general and for LSDs specifically, is enzyme enhancement with small molecule pharmacological chaperones $(28,29)$. Pharmacological chaperone therapy is based on the concept that loss-of-function diseases are often due to missense mutations that cause misfolding (abnormal conformation) of mutant proteins. The misfolded proteins are recognized by the quality control systems of the endoplasmic reticulum and are degraded Thus, in these so-called 'misfolding protein diseases' the loss of function is not due to the loss of catalytic activity, but is the result of the degradation of the aberrant protein. It has been shown that small-molecule ligands (pharmacological chaperones) can interact with the mutant protein, favor its correct conformation, and enhance its stability. As a result, the enzymatic activity of the mutant protein is partially rescued (Fig. 4). As with ERT and other approaches directed toward replacing or increasing the residual activity of the defective enzyme, it is reasonable to speculate that even minor increases in activity may have a favorable impact on patient status and rate of disease progression.

The use of pharmacological chaperones for the treatment of lysosomal storage diseases was first proposed for Fabry disease (30). In cells from patients with Fabry disease, one of the most potent inhibitors of $\alpha$-gal A, 1-deoxygalactonojirimycin (DGJ), was paradoxically shown to enhance residual $\alpha$-galactosidase A activity. Oral administration of DGJ to transgenic mice overexpressing a mutant $\alpha$-galactosidase $\mathrm{A}$ substantially elevated the enzyme activity in some organs (31).

The same approach was subsequently investigated for a restricted number of other disorders of this group $(28,29)$, including Gaucher disease (32) and Pompe disease $(33,34)$.

In principle, chaperones (as with substrate reducing drugs and small molecule drugs in general) have several advantages, as compared to ERT, as they can be administered orally, allowing for a non-invasive treatment, are non-immunogenic and do not need to be delivered to cells through the mannose-6-phosphate pathway, which may be secondarily impaired in some of these disorders. In addition, small molecule chaperones are expected to diffuse freely across cell membranes and reach therapeutic concentrations in different tissues and systems, including the CNS. In the animal model of $\mathrm{G}_{\mathrm{M} 1}$ gangliosidosis, a severe neurodegenerative disorder due to the deficiency of $\beta$-galactosidase, the use of the chaperone $\mathrm{N}$-octyl 4-epi- $\beta$-valienamine (NOEV) resulted in increased residual activity in the brain and greater clearance of substrate (35). 
On the other hand, chaperone therapy appears to be feasible only in patients with responsive mutations mostly located in specific domains of the enzymatic protein (36).

Concerns have also been raised by the fact that most chaperones thus far identified for the treatment of LSDs are active site-directed molecules and, in other words, potential competitive inhibitors of the target enzymes.

These limitations may be overcome by the discovery of new molecules interacting with other protein domains. Recently, allosteric chaperones of $\alpha$-glucosidase, the enzyme deficient in Pompe disease, were identified by the combination of biochemical characterization and a computational analysis of the interactions of these drugs with the enzyme (37). High throughput screenings of chemical libraries may also be a time-efficient way to identify new-generation chaperones $(38,39)$.

After the in vitro studies, suggesting a potential of pharmacological chaperones for the treatment of LSDs, research is now moving into clinical translation. Phase I/II clinical trials are being conducted for Fabry, Gaucher and Pompe disease. For Fabry disease, a phase II/III clinical trial has been performed and has shown encouraging results in terms of enhancement of the residual $\alpha$-galactosidase A enzyme activity and clearance of substrate.

\section{Combination of chaperones and ERT}

Although pharmacological chaperone therapy (PCT) has been developed as a strategy to rescue mutant enzymes from degradation, recent studies showed that chaperones are also able to enhance physical stability and potentiate the therapeutic action of the enzymes used for ERT (40-43). These studies, performed in cell systems and in the animal models of Pompe and Fabry disease, suggested a major change in the use of PCT. In both disorders, when recombinant enzymes were administered in combination with the chaperone molecules, the lysosomal trafficking, the maturation and the intracellular activity of the recombinant enzymes was highly improved.

Although the mechanism underlying this synergistic effect of ERT and pharmacological chaperones is not yet fully understood, this synergy may have important advantages compared to the 'traditional' use of chaperones to rescue mutated enzymes. First, the therapeutic effect is directed toward the exogenous wild-type enzyme used for ERT, it is mutationindependent, and may thus be exploited in any patient on ERT $(37,41)$. Second, while the enhancement of endogenous defective enzymes by chaperones in most cases results in minor changes in terms of residual activity (possibly with a modest impact on patient outcome), the synergy between chaperones and ERT apparently induces, at least in cellular models, notable increases in specific activity with complete or near-complete correction of the enzymatic defect.

Also, these studies suggest that, in principle, the synergistic effect of chaperone therapy and ERT may be seen in any LSD for which ERT and a chaperone are available.

Clinical trials based on the combination of chaperones and ERT are already in progress (see trials NCT00214500 and NCT01196871 at http://clinicaltrials.gov; Telethon foundation trial GUP09017, http://www.telethon.it/ricerca-progetti/ progetti-finanziati).

\section{Proteostasis regulators}

In addition to chaperone therapy, other approaches have been proposed that are aimed at rescuing the mutated enzymes in LSDs. While chaperones are ligands that specifically interact with mutant proteins (and are thus able to rescue a single protein), other drugs are able to adjust the cellular mechanisms controlling the homeostasis of proteins, or proteostasis. Proteostasis is a complex network that controls protein synthesis, folding, trafficking, aggregation and degradation. These small molecule drugs, known as proteostasis regulators, can adjust the capacity of this network and have the potential to restore the normal balance between protein folding, trafficking, and degradation. Two proteostasis regulators have been reported to restore the function of two mutant lysosomal enzymes in two distinct LSDs, Gaucher disease and $\mathrm{G}_{\mathrm{M} 2}$ gangliosidosis (44). Co-administration of a pharmacological chaperone and a proteostasis regulator exhibited synergy and resulted in further enhancement of enzyme activity. This approach remains largely experimental.

\section{Gene therapy}

Gene therapy is an attractive strategy that holds great promise for LSD patients and is commonly viewed as the approach that has the greatest potential for complete and sustained correction of the enzymatic defect $(45,46)$. Gene therapy is also an excellent example of a strategy that is being developed owing to the combination of improved knowledge of the molecular bases of LSDs and technical advancements in the optimization and preparation of therapeutic agents.

Gene therapy, as with the approaches mentioned above, is aimed at increasing or restoring the activity of the defective enzyme in the patient's cells. This is not obtained by supplying the missing enzymatic protein, but by delivering the normal copy of the defective gene, that will direct the synthesis of the normal enzyme by the recipient's cells.

LSDs appear to be amenable to this therapy for several reasons. First, these disorders are monogenic and it is theoretically possible to correct the disease by rectifying the gene defect; second, if lysosomal enzymes are secreted from a small number of transduced cells they can be taken up, via the mannose-6-phosphate receptors, by adjacent affected cells. Such cross-correction, similar to that documented in HSCT, avoids the necessity of transferring the gene to all cells, with the advantage that a small percentage of transduced cells could act therapeutically with consequent benefits for several organs. Third, as already mentioned, even minor increases of enzymatic activity (up to $10 \%$ ) may be sufficient for clinical benefit and partial phenotypic correction.

Different viral vectors and different strategies have been explored to accomplish in vivo gene transfer. Herpesviruses, lentiviruses, adeno-associated viruses (AAVs), adenoviruses (Ads) and others have been tested as vectors (45).

Genetic modification may be performed either ex vivo or in vivo (Fig. 5). The first strategy is based on ex vivo modification of cells and transplantation of the modified cells into patients. Cells that are most commonly considered therapeutic targets for LSDs are hematopoietic progenitor cells. 


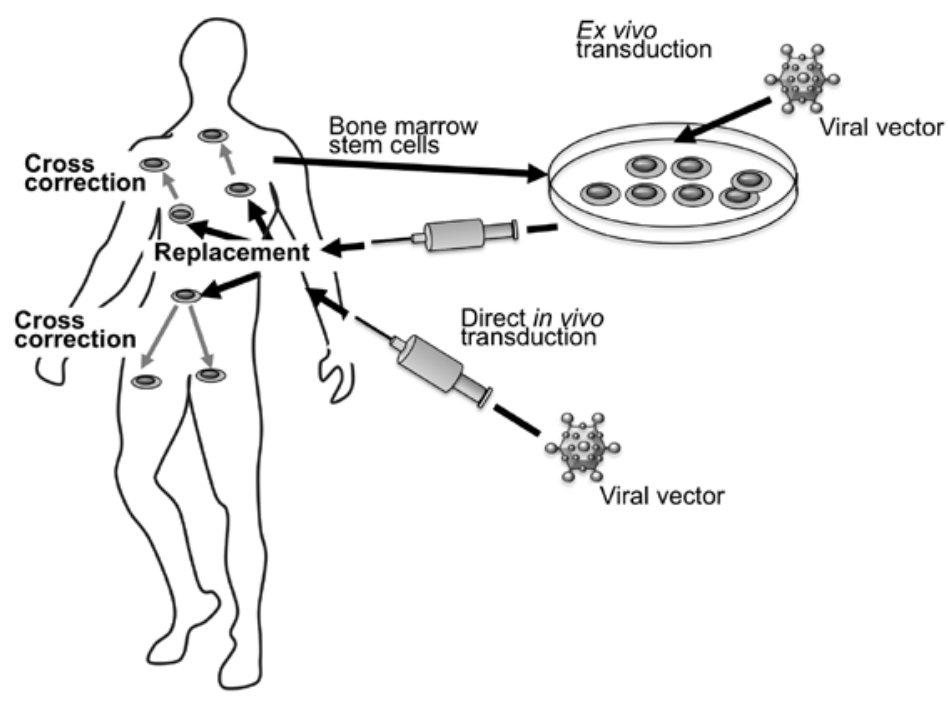

Figure 5. Gene therapy is based on the delivery of the normal copy of the defective gene, that will direct the synthesis of the normal enzyme by the recipient's cells. The genetic modification may be performed by modifying the cells ex vivo, and by transplantation of the modified cells into patients. Another strategy is based on the direct in vivo gene therapy. This approach is based on the injection of a gene transfer vector directly into a tissue or into the circulation. In both cases, transduced cells may either repopulate specific tissues or cross the recipient cells, as shown for HSCT.

Another strategy that is being extensively tested is based on the direct in vivo gene therapy. This approach is based on the injection of a gene transfer vector directly into a tissue or into the circulation.

An advantage of a gene therapy approach compared to ERT is the potential for long-term expression of the therapeutic protein. Thus, this would be a once-and-for-all procedure, and may have obvious advantages compared to ERT and small molecule therapies, that require life-long treatments. Another advantage is that gene therapy may be available to patients with conditions so rare that developing enzyme for ERT is not commercially feasible.

Although gene therapy is a promising treatment for LSDs, some concerns remain. The major issue is viral vector safety and the possibility of carcinogenesis following retrovirus- or adenovirus-mediated gene transfer. Unlike retroviral vectors, AAV vectors are nonpathogenic and generally do not integrate into the host genome and are, in this respect, safer. A second consideration for gene replacement for the LSDs is that the vectors generally express supra-physiologic levels of enzyme and it is not known whether this would be safe in humans.

Other issues are related to the doses of vectors to be used, to the choice of the best vectors to obtain the desired correction of tissue and organs, and to the possibility of immune response toward the wild-type enzyme.

Although cross-correction makes it possible to achieve widespread enzyme distribution while genetically modifying a minority of cells, effective treatment of CNS disease remains an unsolved issue. This is due to the presence of the BBB, which excludes exogenous lysosomal enzymes from the brain. For neuronopathic LSDs, intracerebral injection of the vector carrying the wild-type gene has been explored as a possible strategy. In the animal model of multiple sulfatase deficiency (MSD), a severe autosomal recessive disease is caused by mutations in the sulfatase modifying factor 1 gene (Sumf1), the combination of systemic and intracerebral delivery of the wild-type gene resulted in the global activation of sulfatases, near-complete clearance of glycosaminoglycans, and improved behavioral abilities (47).

Gene therapy is under study for a variety of diseases, mostly in preclinical studies performed in animal models recapitulating the LSDs, phenotypes. The first clinical trials are now in progress or are being planned.

\section{Substrate reduction therapy}

SRT is based on the concept that the inhibition of specific steps of the biosynthetic pathways of substrates may reduce their flux to lysosomes and help restore the equilibrium between their synthesis and catabolism $(48,49)$. This task is generally accomplished by using small-molecule inhibitors of enzymes involved in the biosynthesis of substrates.

SRT has already been approved for clinical use for the treatment of type 1 Gaucher disease, the most prevalent LSD, due to $\beta$-glucocerebrosidase deficiency and characterized by glycosphingolipid storage, and of Niemann-Pick disease type C (NPC), a defect of intracellular cholesterol trafficking.

The largest clinical experience on this approach has been obtained with the use of N-butyldeoxynojirimycin (Miglustat) in Gaucher disease. In this disorder, Miglustat has been shown to be effective in improving or stabilizing biochemical, visceral, hematologic and bone markers of the disease (50), causing limited adverse events (51).

A novel substrate inhibitor, eliglustat tartrate, was recently introduced and evaluated in a multisite, open-label, single-arm phase II clinical trial (52). Treatment with eliglustat tartrate resulted in improved hemoglobin levels and platelet counts, reduced spleen and liver volume, and increased lumbar spine bone mineral density.

In NPC, it has been demonstrated that glycosphingolipid storage plays a pathogenic role in brain disease (53). Thus, it was proposed that Miglustat, the same therapeutic agent used for Gaucher disease, could also be beneficial in NPC patients. 
In a controlled trial in 29 adult and juvenile NPC patients, Miglustat stabilized neurological disease, with improved horizontal saccadic eye movement, stabilized ambulation, and improved swallowing $(54,55)$. Improved swallowing was also observed in a study on the efficacy of Miglustat in four pediatric patients (56).

The approach based on SRT has also been extended to the treatment of MPS. Preclinical and clinical studies were based on the use of non-specific inhibitors of glycosaminoglycan synthesis, the isoflavone genistein and the chemical dye rhodamine B (57-60). The effects of these drugs, however, have been quite variable so far. In a two-year follow-up study of eight MPS III patients (61) treated with genistein, five patients showed improvement or stabilization of cognitive functions, whereas three showed further deterioration.

SRT has also been proposed in a few preclinical studies for the treatment of other LSDs, such as Sandhoff disease (62), Fabry disease (63), and Pompe disease $(64,65)$.

The use of small-molecule inhibitors of substrate synthesis has obvious advantages, compared to the use of ERT and gene therapy, particularly in terms of bioavailability in different tissues and organs, including the CNS. The efficacy profile in NPC and other disorders, however, remains to be fully defined.

\section{Other experimental approaches}

The improvement of the knowledge on the molecular bases of genetic diseases and on the pathophysiology of LSDs has recently indicated additional targets of therapy.

As an example, it has been shown that manipulation of the autophagic pathway may have a beneficial effect in the animal model of Pompe disease. Genetic suppression of autophagy in the Pompe mouse resulted in reduced glycogen accumulation in skeletal muscle by $50-60 \%$ compared with mice with genetically intact autophagy (66). In addition, in the autophagy-defective Pompe animals, the efficacy of ERT was greatly improved with a near-complete clearance of glycogen in the skeletal muscle, a therapeutic goal never attained in PD mice with genetically intact autophagy.

Therapeutic strategies have been developed to induce ribosomal read-through of nonsense mutations in mRNA and allow production of a full-length functional protein. Small-molecule drugs such as aminoglycosides and ataluren (PTC124) have been developed and are in clinical testing in patients with several genetic disorders, such as Becker/Duchenne muscular dystrophy, cystic fibrosis, methylmalonic acidemia and others (67-70). In principle, use of nonsense mutation suppression may offer the prospect of treating patients with other genetic diseases due to premature termination of ribosomal reading, including LSDs. This approach has been explored in preclinical studies in cells from neuronal lipofuscinoses due to palmitoyl-thioesterase deficiency (71).

An entirely new and attractive concept of treatment derived from recent discoveries on the biology of lysosomes. It has been shown that lysosomal biogenesis and expression is regulated by the transcription factor EB (TFEB) (72). TFEB also regulates lysosomal exocytosis (73) by increasing the pool of lysosomes in the periphery of cells and facilitating their fusion with the plasma membrane. Induction of lysosomal exocytosis by TFEB overexpression rescued pathological storage and restored normal cellular morphology in vitro in cells from different LSDs (Pompe disease, multiple sulfatase deficiency, mucopolysaccharidosis type IIIA) and in vivo in the animal model of multiple sulfatase deficiency. These results indicated an alternative therapeutic strategy for disorders associated with intracellular storage, based on stimulation of lysosomal exocytosis.

\section{Conclusion}

The past two decades have given rise to significant progress in the treatment of LSDs. Different therapeutic options are now available or are being evaluated in preclinical studies. However, none of these options is applicable to all lysosomal diseases and most of the approaches have important limitations related to the bioavailability of therapeutic agents, toxicity, immune response, and impact on patient quality of life.

Future research is required to address these issues. Particular attention should be paid to the clear definition of guidelines for the treatment of patients. In this respect, careful collection of information in international registries on the natural history of LSDs and on the effects of therapies will be beneficial.

The possibility to combine different approaches in order to obtain the highest therapeutic efficacy and to personalize treatment protocols for each disorder and for each individual patient must be explored.

\section{References}

1. Futerman AH and van Meer G: The cell biology of lysosomal storage disorders. Nat Rev Mol Cell Biol 5: 554-565, 2004.

2. Fuller M, Meikle PJ and Hopwood JJ: Epidemiology of lysosomal storage diseases: an overview. In: Fabry Disease: Perspectives from 5 years of FOS. Mehta A, Beck M and Sunder-Plassmann G (eds). Oxford PharmaGenesis, Oxford, 2006.

3. Beck M: New therapeutic options for lysosomal storage disorders: enzyme replacement, small molecules and gene therapy. Hum Genet 121: 1-22, 2007.

4. Beutler E: Lysosomal storage diseases: natural history and ethical and economic aspects. Mol Genet Metab 88: 208-215, 2006.

5. Ballabio A and Gieselmann V: Lysosomal disorders: from storage to cellular damage. Biochim Biophys Acta 1793: 684-696, 2009.

6. Prasad VK and Kurtzberg J: Transplant outcomes in mucopolysaccharidoses. Semin Hematol 47: 59-69, 2010.

7. Valayannopoulos V and Wijburg FA: Therapy for the mucopolysaccharidoses. Rheumatology (Oxford) 50 (Suppl 5): v49-v59, 2011.

8. Orchard PJ, Blazar BR, Wagner J, Charnas L, Krivit W and Tolar J: Hematopoietic cell therapy for metabolic disease. J Pediatr 151: 340-346, 2007.

9. de Ru MH, Boelens JJ, Das AM, Jones SA, van der Lee JH, Mahlaoui N, Mengel E, Offringa M, O'Meara A, Parini R, Rovelli A, Sykora KW, Valayannopoulos V, Vellodi A, Wynn RF and Wijburg FA: Enzyme replacement therapy and/or hematopoietic stem cell transplantation at diagnosis in patients with mucopolysaccharidosis type I: results of a European consensus procedure. Orphanet J Rare Dis 6: 55, 2011.

10. Orchard PJ and Tolar J: Transplant outcomes in leukodystrophies. Semin Hematol 47: 70-78, 2010.

11. Sly WS: Receptor-mediated transport of acid hydrolases to lysosomes. Curr Top Cell Regul 26: 27-38, 1985.

12. Barton NW, Brady RO, Dambrosia JM, Di Bisceglie AM, Doppelt SH, Hill SC, Mankin HJ, Murray GJ, Parker RI, Argoff CE, et al: Replacement therapy for inherited enzyme deficiency - macrophage-targeted glucocerebrosidase for Gaucher's disease. N Engl J Med 324: 1464-1470, 1991.

13. Barton NW, Furbish FS, Murray GJ, Garfield M and Brady RO: Therapeutic response to intravenous infusions of glucocerebrosidase in a patient with Gaucher disease. Proc Natl Acad Sci USA 87: 1913-1916, 1990 
14. Mehta A, Beck M, Elliott P, Giugliani R, Linhart A, SunderPlassmann G, Schiffmann R, Barbey F, Ries M and Clarke JT; Fabry Outcome Survey investigators. Enzyme replacement therapy with agalsidase alfa in patients with Fabry's disease: an analysis of registry data. Lancet 374: 1986-1996, 2009.

15. Lidove O, West ML, Pintos-Morell G, Reisin R, Nicholls K, Figuera LE, Parini R, Carvalho LR, Kampmann C, Pastores GM and Mehta A: Effects of enzyme replacement therapy in Fabry disease - a comprehensive review of the medical literature. Genet Med 12: 668-679, 2010

16. Feriozzi S, Torras J, Cybulla M, Nicholls K, Sunder-Plassmann G and West M; FOS Investigators: The effectiveness of long-term agalsidase alfa therapy in the treatment of Fabry nephropathy. Clin J Am Soc Nephrol 7: 60-69, 2012.

17. van der Ploeg AT and Reuser AJ: Pompe's disease. Lancet 372 , $1342-1353,2008$

18. Strothotte S, Strigl-Pill N, Grunert B, Kornblum C, Eger K, Wessig C, Deschauer M, Breunig F, Glocker FX, Vielhaber S, Brejova A, Hilz M, Reiners K, Müller-Felber W, Mengel E, Spranger M and Schoser B: Enzyme replacement therapy with alglucosidase alfa in 44 patients with late-onset glycogen storage disease type 2: 12-month results of an observational clinical trial. J Neurol 257: 91-97, 2010

19. van der Ploeg AT, Clemens PR, Corzo D, Escolar DM, Florence J, Groeneveld GJ, Herson S, Kishnani PS, Laforet P, Lake SL, Lange DJ, Leshner RT, Mayhew JE, Morgan C, Nozaki K, Park DJ, Pestronk A, Rosenbloom B, Skrinar A, van Capelle CI, van der Beek NA, Wasserstein M and Zivkovic SA: A randomized study of alglucosidase alfa in late-onset Pompe's disease. N Engl J Med 362: 1396-1406, 2010.

20. Parenti G and Andria G: Pompe disease: from new views on pathophysiology to innovative therapeutic strategies. Curr Pharm Biotechnol 12: 902-915, 2011.

21. Anson DS, McIntyre C and Byers S: Therapies for neurological disease in the mucopolysaccharidoses. Curr Gene Ther 11: 132-143, 2011.

22. Begley DJ, Pontikis CC and Scarpa M: Lysosomal storage diseases and the blood-brain barrier. Curr Pharm Des 14: 1566-1580, 2008.

23. Grubb JH, Vogler C, Levy B, Galvin N, Tan Y and Sly WS Chemically modified beta-glucuronidase crosses blood-brain barrier and clears neuronal storage in murine mucopolysaccharidosis VII. Proc Natl Acad Sci USA 105: 2616-2621, 2008.

24. Osborn MJ, McElmurry RT, Peacock B, Tolar J and Blazar BR: Targeting of the CNS in MPS-IH using a nonviral transferrin-alpha-L-iduronidase fusion gene product. Mol Ther 16: 1459-1466, 2008.

25. Munoz-Rojas MV, Vieira T, Costa R, Fagondes S, John A, Jardim LB, Vedolin LM, Raymundo M, Dickson PI, Kakkis E and Giugliani R: Intrathecal enzyme replacement therapy in a patient with mucopolysaccharidosis type I and symptomatic spinal cord compression. Am J Med Genet A 146A: 2538-2544, 2008.

26. Zhu Y, Jiang JL, Gumlaw NK, Zhang J, Bercury SD, Ziegler RJ, Lee K, Kudo M, Canfield WM,Edmunds T, Jiang C, Mattaliano RJ and Cheng SH: Glycoengineered acid alpha-glucosidase with improved efficacy at correcting the metabolic aberrations and motor function deficits in a mouse model of Pompe disease. Mol Ther 17: 954-963, 2009.

27. Zimran A, Brill-Almon E, Chertkoff R, Petakov M, BlancoFavela F, Muñoz ET, Solorio-Meza SE, Amato D, Duran G, Giona F, Heitner R, Rosenbaum H, Giraldo P, Mehta A, Park G, Phillips M, Elstein D, Altarescu G, Szleifer M, Hashmueli S and Aviezer D: Pivotal trial with plant cell-expressed recombinant glucocerebrosidase, taliglucerase alfa, a novel enzyme replacement therapy for Gaucher disease. Blood 118: 5767-5773, 2011.

28. Parenti G: Treating lysosomal storage diseases with pharmacological chaperones: from concept to clinics. EMBO Mol Med 1: 268-279, 2009

29. Valenzano KJ, Khanna R, Powe AC, Boyd R, Lee G, Flanagan JJ and Benjamin ER: Identification and characterization of pharmacological chaperones to correct enzyme deficiencies in lysosomal storage disorders. Assay Drug Dev Technol 9: 213-235, 2011.

30. Fan JQ, Ishii S, Asano N and Suzuki Y: Accelerated transport and maturation of lysosomal alpha-galactosidase A in Fabry lymphoblasts by an enzyme inhibitor. Nat Med 5: 112-115, 1999.

31. Germain DP and Fan JQ: Pharmacological chaperone therapy by active-site-specific chaperones in Fabry disease: in vitro and preclinical studies. Int J Clin Pharmacol Ther 47 (Suppl 1): S111-S117, 2009.
32. Sawkar AR, Adamski-Werner SL, Cheng WC, Wong $\mathrm{CH}$, Beutler E, Zimmer KP and Kelly JW: Gaucher disease-associated glucocerebrosidases show mutation-dependent chemical chaperoning profiles. Chem Biol 12: 1235-1244, 2005.

33. Parenti G, Zuppaldi A, Gabriela Pittis M, Rosaria Tuzzi M, Annunziata I, Meroni G, Porto C, Donaudy F, Rossi B, Rossi M, Filocamo M, Donati A, Bembi B, Ballabio A and Andria G: Pharmacological enhancement of mutated alpha-glucosidase activity in fibroblasts from patients with Pompe disease. Mol Ther 15: 508-514, 2007

34. Okumiya T, Kroos MA, Vliet LV, Takeuchi H, Van der Ploeg AT and Reuser AJ: Chemical chaperones improve transport and enhance stability of mutant alpha-glucosidases in glycogen storage disease type II. Mol Genet Metab 90: 49-57, 2007.

35. Suzuki Y, Ichinomiya S, Kurosawa M, Matsuda J, Ogawa S, Iida M, Kubo T, Tabe M, Itoh M, Higaki K, Nanba E and Ohno K: Therapeutic chaperone effect of N-octyl 4-epi- $\beta$-valienamine on murine G(M1)-gangliosidosis. Mol Genet Metab 106: 92-98, 2012.

36. Flanagan JJ, Rossi B, Tang K, Wu X, Mascioli K, Donaudy F, Tuzzi MR, Fontana F, Cubellis MV, Porto C, Benjamin E, Lockhart DJ, Valenzano KJ, Andria G, Parenti G and Do HV: The pharmacological chaperone 1-deoxynojirimycin increases the activity and lysosomal trafficking of multiple mutant forms of acid alpha-glucosidase. Hum Mutat 30: 1683-1692, 2009.

37. Porto C, Ferrara MC, Meli M, Acampora E, Avolio V, Rosa M, Cobucci-Ponzano B, Colombo G, Moracci M, Andria G and Parenti G: Pharmacological enhancement of alpha-glucosidase by the allosteric chaperone N-acetylcysteine. Mol Ther: Sep 18 , 2012 (Epub ahead of print). doi: 10.1038/mt.2012.152.

38. Urban DJ, Zheng W, Goker-Alpan O, Jadhav A, Lamarca ME, Inglese J, Sidransky E and Austin CP: Optimization and validation of two miniaturized glucocerebrosidase enzyme assays for high throughput screening. Comb Chem High Throughput Screen 11: 817-824, 2008

39. Marugan JJ, Zheng W, Motabar O, Southall N, Goldin E, Sidransky E, Aungst RA, Liu K, Sadhukhan SK and Austin CP: Evaluation of 2-thioxo-2,3,5,6,7,8-hexahydropyrimido(4,5-d) pyrimidin- $4(1 \mathrm{H})$-one analogues as GAA activators. Eur J Med Chem 45: 1880-1897, 2010.

40. Porto C, Cardone M, Fontana F, Rossi B, Tuzzi MR, Tarallo A, Barone MV, Andria G and Parenti G: The pharmacological chaperone N-butyldeoxynojirimycin enhances enzyme replacement therapy in Pompe disease fibroblasts. Mol Ther 17: 964-971, 2009

41. Porto C, Pisani A, Rosa M, Acampora E, Avolio V, Tuzzi MR, Visciano B, Gagliardo C, Materazzi S, la Marca G, Andria G and Parenti G: Synergy between the pharmacological chaperone 1-deoxygalactonojirimycin and the human recombinant alphagalactosidase A in cultured fibroblasts from patients with Fabry disease. J Inherit Metab Dis 35: 513-520, 2012.

42. Benjamin ER, Khanna R, Schilling A, Flanagan JJ, Pellegrino LJ, Brignol N, Lun Y, Guillen D, Ranes BE, Frascella M, Soska R, Feng J, Dungan L, Young B, Lockhart DJ and Valenzano KJ: Co-administration with the pharmacological chaperone AT1001 increases recombinant human $\alpha$-galactosidase A tissue uptake and improves substrate reduction in Fabry mice. Mol Ther 20: 717-726, 2012.

43. Khanna R, Flanagan JJ, Feng J, Soska R, Frascella M, Pellegrino LJ, Lun Y, Guillen D, Lockhart DJ and Valenzano KJ: The pharmacological chaperone AT2220 increases recombinant human acid $\alpha$-glucosidase uptake and glycogen reduction in a mouse model of pompe disease. PLoS One 7: e40776, 2012.

44. Mu TW, Ong DS, Wang YJ, Balch WE, Yates JR III, Segatori L and Kelly JW: Chemical and biological approaches synergize to ameliorate protein-folding diseases. Cell 134: 769-781, 2008.

45. Sands MS and Davidson BL: Gene therapy for lysosomal storage diseases. Mol Ther 13: 839-849, 2006

46. Cardone M: Prospects for gene therapy in inherited neurodegenerative diseases. Curr Opin Neurol 20: 15-18, 2007.

47. Spampanato C, De Leonibus E, Dama P, Gargiulo A, Fraldi A, Sorrentino NC, Russo F, Nusco E, Auricchio A, Surace EM and Ballabio A: Efficacy of a combined intracerebral and systemic gene delivery approach for the treatment of a severe lysosomal storage disorder. Mol Ther 19: 860-869, 2011.

48. Platt FM and Jeyakumar M: Substrate reduction therapy. Acta Paediatr (Suppl) 97: 88-93, 2008.

49. Schiffmann R: Therapeutic approaches for neuronopathic lysosomal storage disorders. J Inherit Metab Dis 33: 373-379, 2010. 
50. Giraldo P, Alfonso P, Atutxa K, Fernández-Galán MA, Barez A Franco R, Alonso D, Martin A, Latre P and Pocovi M: Real-world clinical experience with long-term miglustat maintenance therapy in type 1 Gaucher disease: the ZAGAL project. Haematologica 94: 1771-1775, 2009.

51. Hollak CE, Hughes D, van Schaik IN, Schwierin B and Bembi B: Miglustat (Zavesca) in type 1 Gaucher disease: 5-year results of a post-authorisation safety surveillance programme. Pharmacoepidemiol Drug Saf 18: 770-777, 2009.

52. Lukina E, Watman N, Arreguin EA, Dragosky M, Iastrebner M, Rosenbaum H, Phillips M, Pastores GM, Kamath RS, Rosenthal DI, Kaper M, Singh T, Puga AC and Peterschmitt MJ Improvement in hematological, visceral, and skeletal manifestations of Gaucher disease type 1 with oral eliglustat tartrate (Genz-112638) treatment: 2-year results of a phase 2 study. Blood 116: 4095-4098, 2010.

53. Lachmann RH, te Vruchte D, Lloyd-Evans E, Reinkensmeier G, Sillence DJ, Fernandez-Guillen L, Dwek RA, Butters TD, Cox TM and Platt FM: Treatment with miglustat reverses the lipid-trafficking defect in Niemann-Pick disease type C. Neurobiol Dis 16: 654-658, 2004.

54. Patterson MC, Vecchio D, Prady H, Abel L and Wraith JE: Miglustat for treatment of Niemann-Pick C disease: a randomised controlled study. Lancet Neurol 6: 765-772, 2007.

55. Wraith JE, Vecchio D, Jacklin E, Abel L, Chadha-Boreham H, Luzy C, Giorgino R and Patterson MC: Miglustat in adult and juvenile patients with Niemann-Pick disease type C: long-term data from a clinical trial. Mol Genet Metab 99: 351-357, 2010.

56. Fecarotta S, Amitrano M, Romano A, Della Casa R, Bruschini D, Astarita L, Parenti G and Andria G: The videofluoroscopic swallowing study shows a sustained improvement of dysphagia in children with Niemann-Pick disease type $\mathrm{C}$ after therapy with miglustat. Am J Med Genet A 155A: 540-547, 2011.

57. Piotrowska E, Jakóbkiewicz-Banecka J, Barańska S, TylkiSzymańska A, Czartoryska B, Wegrzyn A and Wegrzyn G: Genistein-mediated inhibition of glycosaminoglycan synthesis as a basis for gene expression-targeted isoflavone therapy for mucopolysaccharidoses. Eur J Hum Genet 14: 846-852, 2006.

58. Roberts AL, Thomas BJ, Wilkinson AS, Fletcher JM and Byers S: Inhibition of glycosaminoglycan synthesis using rhodamine B in a mouse model of mucopolysaccharidosis type IIIA. Pediatr Res 60: 309-314, 2006.

59. Roberts AL,Fletcher JM, Moore L and Byers S: Trans-generational exposure to low levels of rhodamine B does not adversely affect litter size or liver function in murine mucopolysaccharidosis type IIIA. Mol Genet Metab 101: 208-213, 2010.

60. Malinowska M, Wilkinson FL, Langford-Smith KJ, Langford-Smith A, Brown JR, Crawford BE, Vanier MT, Grynkiewicz G, Wynn RF, Wraith JE, Wegrzyn G and Bigger BW: Genistein improves neuropathology and corrects behaviour in a mouse model of neurodegenerative metabolic disease. PLoS One 5: e14192, 2010.

61. Piotrowska E, Jakobkiewicz-Banecka J, Maryniak A, TylkiSzymanska A, Puk E, Liberek A, Wegrzyn A, Czartoryska B, Slominska-Wojewodzka M and Wegrzyn G: Two-year follow-up of Sanfilippo disease patients treated with a genistein-rich isoflavone extract: assessment of effects on cognitive functions and general status of patients. Med Sci Monit 17: 196-202, 2011.
62. Ashe KM, Bangari D, Li L, Cabrera-Salazar MA, Bercury SD, Nietupski JB, Cooper CG, Aerts JM, Lee ER, Copeland DP, Cheng SH, Scheule RK and Marshall J: Iminosugar-based inhibitors of glucosylceramide synthase increase brain glycosphingolipids and survival in a mouse model of Sandhoff disease. PLoS One 6: e21758, 2011.

63. Marshall J, Ashe KM, Bangari D, McEachern K, Chuang WL, Pacheco J, Copeland DP, Desnick RJ, Shayman JA, Scheule RK and Cheng SH: Substrate reduction augments the efficacy of enzyme therapy in a mouse model of Fabry disease. PLoS One 5: e15033, 2010

64. Douillard-Guilloux G, Raben N, Takikita S, Batista L, Caillaud C and Richard E: Modulation of glycogen synthesis by RNA interference: towards a new therapeutic approach for glycogenosis type II. Hum Mol Genet 17: 3876-3886, 2008.

65. Douillard-Guilloux G, Raben N, Takikita S, Ferry A, Vignaud A, Guillet-Deniau I, Favier M, Thurberg BL, Roach PJ, Caillaud C and Richard E: Restoration of muscle functionality by genetic suppression of glycogen synthesis in a murine model of Pompe disease. Hum Mol Genet 19: 684-696, 2010.

66. Raben N, Schreiner C, Baum R, Takikita S, Xu S, Xie T, Myerowitz R, Komatsu M, Van der Meulen JH, Nagaraju K, Ralston E and Plotz PH: Suppression of autophagy permits successful enzyme replacement therapy in a lysosomal storage disorder - murine Pompe disease. Autophagy 6: 1078-1089, 2010

67. Jones AM and Helm JM: Emerging treatments in cystic fibrosis. Drugs 69: 1903-1910, 2009

68. Nelson SF, Crosbie RH, Miceli MC, Spencer MJ: Emerging genetic therapies to treat Duchenne muscular dystrophy. Curr Opin Neurol 22: 532-538, 2009.

69. Sermet-Gaudelus I, De Boeck K, Casimir GJ, Vermeulen F, Leal T, Mogenet A, Roussel D, Fritsch J, Hanssens L, Hirawat S, Miller NL, Constantine S, Reha A, Ajayi T, Elfring GL and Miller LL: Ataluren (PTC124) induces CFTR protein expression and activity in children with nonsense mutation cystic fibrosis. Am J Respir Crit Care Med 182: 1262-1272, 2010.

70. Finkel RS: Read-through strategies for suppression of nonsense mutations in Duchenne/Becker muscular dystrophy: aminoglycosides and ataluren (PTC124). J Child Neurol 25: 1158-1164, 2010.

71. Sarkar C, Zhang Z and Mukherjee AB: Stop codon read-through with PTC124 induces palmitoyl-protein thioesterase-1 activity, reduces thioester load and suppresses apoptosis in cultured cells from INCL patients. Mol Genet Metab 104: 338-345, 2011.

72. Medina DL, Fraldi A, Bouche V, Annunziata F, Mansueto G, Spampanato C, Puri C, Pignata A, Martina JA, Sardiello M, Palmieri M, Polishchuk R, Puertollano R and Ballabio A: Transcriptional activation of lysosomal exocytosis promotes cellular clearance. Dev Cell 21: 421-430, 2011.

73. Sardiello M, Palmieri M, di Ronza A, Medina DL, Valenza M, Gennarino VA, Di Malta C, Donaudy F, Embrione V, Polishchuk RS, Banfi S, Parenti G, Cattaneo E and Ballabio A: A gene network regulating lysosomal biogenesis and function. Science 325 : 473-477, 2009. 